\title{
Water-responsive shape memory hybrid: Design concept and demonstration
}

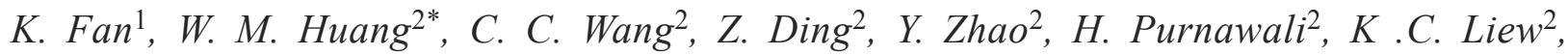 \\ L. X. Zheng ${ }^{2}$ \\ ${ }^{1}$ Hwa Chong Institution, 661 Bukit Timah Road, 269734 Singapore \\ ${ }^{2}$ School of Mechanical and Aerospace Engineering, Nanyang Technological University, 50 Nanyang Avenue, 639798 \\ Singapore
}

Received 9 September 2010; accepted in revised form 23 November 2010

\begin{abstract}
Shape memory materials are featured by their ability to recover their original shapes when a particular stimulus, such as heat, light, magnetic field, even moisture/water, etc. is applied. However, it is not an easy task for non-professionals to synthesize a shape memory material which can meet all the requirements of a particular application. Even for professionals, like materials researchers, it could involve tedious trial and error procedures. In this paper, the concept of water-responsive shape memory hybrid is proposed and the advantages are demonstrated by two examples. The hybrid concept is versatile and can be easily accessed by those even without much polymer/chemistry background. Moreover, the performance of such hybrids can be well-predicted. This concept can be further extended into solvent-responsive shape memory hybrids, which can be routinely designed and realized in a Do-It-Yourself manner by almost anyone.
\end{abstract}

Keywords: Tailor-made polymers, smart polymers, polymer composites

\section{Introduction}

Shape memory materials are featured by their ability to recover their original shapes when a particular stimulus is applied [1]. For shape memory alloys (SMAs), the stimuli are thus far limited to heat (including joule heating) and magnetic field only [2-4], whereas for shape memory polymers (SMPs), the stimuli are further extended to include light, chemical (including water, $\mathrm{pH}$ change, etc) and many others [5-8].

Shape memory materials, in particular SMAs, have been used in a wide range of engineering applications for many years $[2,3,9]$. More recently, SMPs have been attracting a lot of attention due to their great potential, in particular for biomedical applications [10-12]. As compared with SMAs [13], SMPs are lower in cost (for both material and processing), and easier to be customized to achieve tailored properties and functions to meet the needs of a particular application [2, 11, 14-18].

Despite these advantages, the synthesis of a new SMP requires not only strong background in polymer/chemistry but also extensive trial and error procedures. Moreover, although there are a myriad number of SMPs that have been developed or still under development all over the world, till today few of these SMPs are commercially available or widely accepted in some specific fields. One of the rationales behind it is that it is relatively easy to develop a new SMP, but as a new material, it requires tremendous effort and time to conduct various tests in order to ensure its reliability/stability for any real engineering applications [19-21]. For instance, long term stability of materials, such as SMP stents [22],

\footnotetext{
${ }^{*}$ Corresponding author, e-mail: mwmhuang@ntu.edu.sg
}

(c) BME-PT 
within a liquid environment is a major concern in the biomedical applications.

From the application point of view, the ideal solution is to use the materials that have been traditionally used in the industry, so that devices made of these materials are not only compatible (in every aspect) to both the fabrication processes and the working environment but also meets the particular requirements. Furthermore, its performance can also be well-predicted even in the early design stage.

In this paper, a hybrid concept was demonstrated to achieve water-driven shape recovery, i.e., waterresponsive shape memory hybrid was designed and produced. This concept is generic and easily accessible to those even without much chemistry/polymer knowledge.

It should also be pointed out that the focus of this paper is twofold, one is for proof of concept and the other is for demonstrating the convenience and versatility in the design and fabrication of such shape memory materials in a Do-It-Yourself manner.

\section{Mechanism and concept}

Unlike SMAs, in which the reversible martensitic transformation is the driving mechanism behind the shape memory phenomenon [2], the shape memory effect in polymers is the result of a two-segment system [1]. As illustrated in Figure 1a, a SMP normally includes an elastic segment, which is always elastic within the temperature range of our interest, and a transition segment, which is able to dramatically alter its stiffness when a particular stimulus, which might be heat, light or water etc., is applied. At the presence of the right stimulus, the transition segment becomes soft, so that the polymer can be easily stretched, while the elastic segment is also deformed accordingly (Figure $1 \mathrm{~b}$ ). Upon removal of the stimulus, the transition segment becomes hard again and thus, the deformation of the elastic

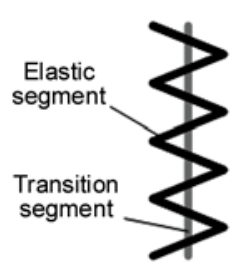

(a)

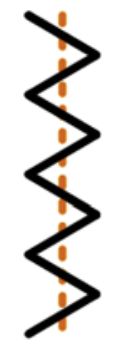

(b)

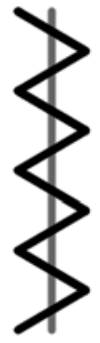

(c)

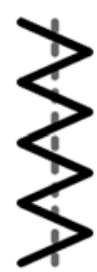

(d)
Figure 1. Illustration of the shape memory effect: normal state (a), deformed state (b), preserved state (c), recovered state $(d)$ segment is largely preserved (Figure 1c). The polymer can virtually maintain this temporary shape (stretched shape) forever. Only the presence of a stimulus (either the same stimulus as previously used for fixing the temporary shape or a different one) that causes the softening of the transition segment can trigger the release of the stored elastic energy in the elastic segment. Consequently, the polymer recovers its original shape (Figure 1d).

This mechanism for the shape memory effect in polymers can also be applied into hybrids, resulting in the avoidance of the difficulties in the syntheses of polymers, which require not only strong background knowledge and extensive experience in polymer/chemistry but also tremendous efforts in trial and error procedures. Fundamentally, such a hybrid is a kind of composite, consisting of matrix (the elastic segment) and inclusion (the transition segment), similar to the mixtures reported in [23]. By selecting the right materials for the matrix and inclusion, together with the right processing technique, we can minimize the chemical interaction between the elastic segment and transition segment as much as possible, so that the properties and behaviors of the resulted materials can be well predicted from the very beginning.

Water (or moisture)-responsive SMP has been realized in a couple of polymer systems [24, 25]. Because of the strong interaction between the elastic and transition segments in these polymers, it is difficult to predict their properties (such as the transition/ shape recovery temperature and stiffness) and behaviors (e.g. the recovery stress and recoverable strain). Individual characterization of their respective properties/behaviors after synthesis is required. As such, tailoring a shape memory material to meet the requirements of a particular application, which is possible in theory, is in fact a tedious and costly process in practice.

In the next section, based on the concept of shape memory hybrid discussed above, we demonstrate two simple approaches to achieve water-responsive shape memory hybrids, in which the chemical interaction between the elastic and transition segments is largely avoided.

\section{Fabrication and demonstration}

As discussed above, the chemical interaction between the elastic and transition segments in shape memory 
hybrids should be minimized or totally avoided (if possible), so that the properties and behaviors of the resulted hybrids can be well predicted from the very beginning. This can be achieved in two ways. One is to select the material components (for the elastic matrix and the transition inclusion) which do not have any possible chemical reaction at all, while the other is to select a processing technique to avoid or minimize such chemical reaction.

Here, two examples of the preparation of waterresponsive shape memory hybrids using two different approaches are presented. To achieve the waterresponsive function in the simplest way, a solid material that can dissolve in the water is used as the transition inclusion. This material should be adequately strong and be in the powder/micro particle form for easy and good dispersion within the elastic matrix. Many non-organic crystals, such as cupric sulphate and sodium acetate, are water-soluble. They are good candidates, at least for demonstration purpose at this stage. Whereas for the elastic matrix, the material should be elastic and stable throughout the entire working processes.

Depending on the material properties of the elastic matrix and transition inclusion, different processing procedures can be applied to fabricate a shape memory hybrid.

\subsection{Polymer sponge/cupric sulphate pentahydrate hybrid}

Cupric sulphate pentahydrate bought from SigmaAldrich, was used as the transition inclusion. The elastic matrix was a polymer sponge, which was selected mainly because it can be significantly compressed. An aluminum cone (base diameter: $2 \mathrm{~cm}$; height: $5 \mathrm{~cm})$ was pushed through the sponge $(2.8 \mathrm{~cm}$ long $\times 2.5 \mathrm{~cm}$ wide $\times 1.3 \mathrm{~cm}$ thick) along its thickness, as shown in Figure 2a and b. Figure 2c shows a small through-thickness crack after the removal of the cone, indicating that a permanent deformation, although limited, did occur.

The water-responsive feature was demonstrated by means of showing the closure of a large hole inside the sponge upon immersing into room temperature water (about $22^{\circ} \mathrm{C}$ ).

The experiment was done in two steps. The details of the first step are as follows.

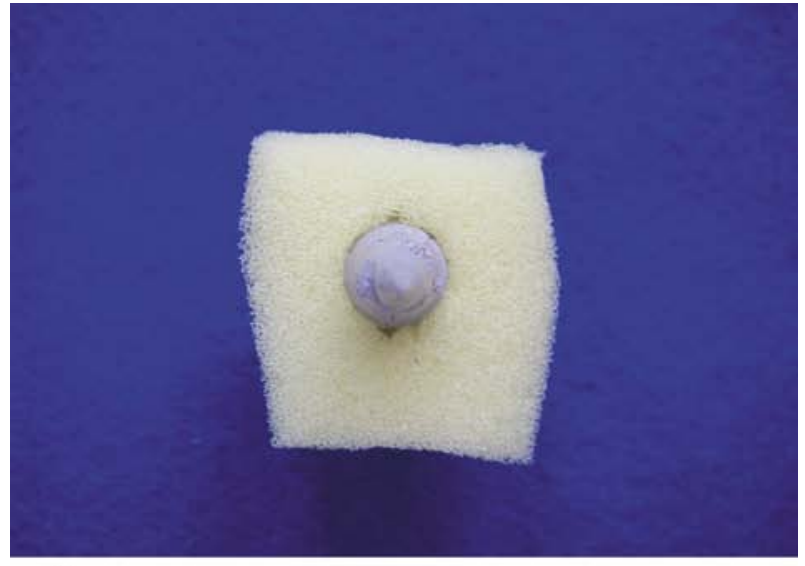

a)

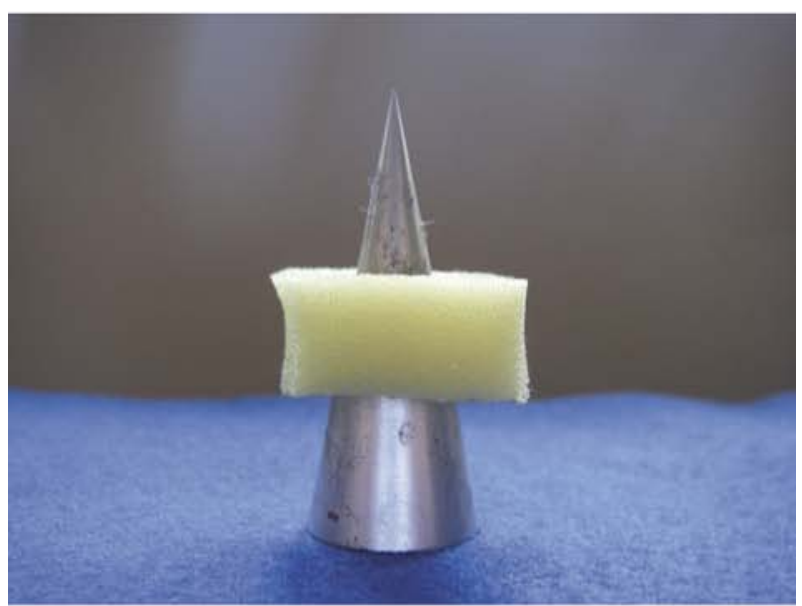

b)

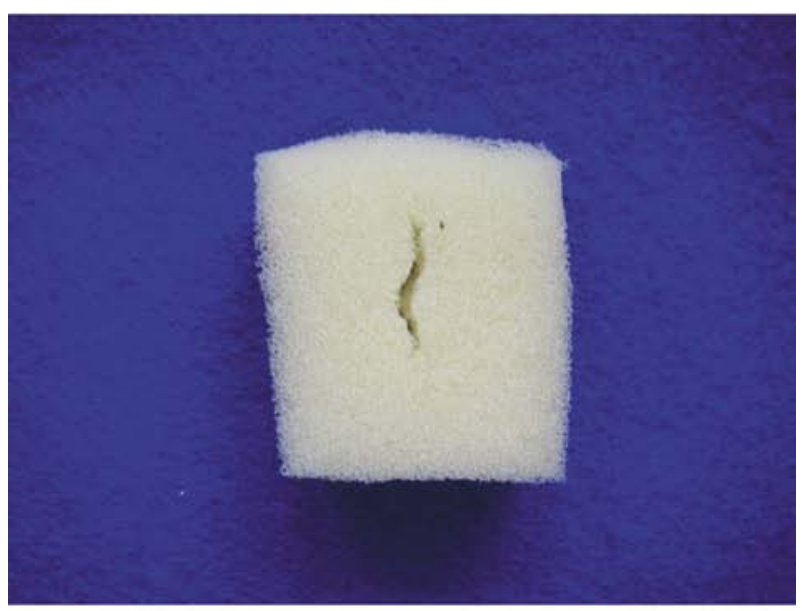

c)

Figure 2. Polymer sponge with a sharp end aluminum cone punched through. Top view (a), side view (b), after removal of aluminum cone (c).

1. A saturated solution of cupric sulfate was prepared in a ratio of $20 \mathrm{~g}$ cupric sulfate pentahydrate per $100 \mathrm{ml}$ of water.

2. The solution was heated to $100^{\circ} \mathrm{C}$ to accelerate the dissolution process. 


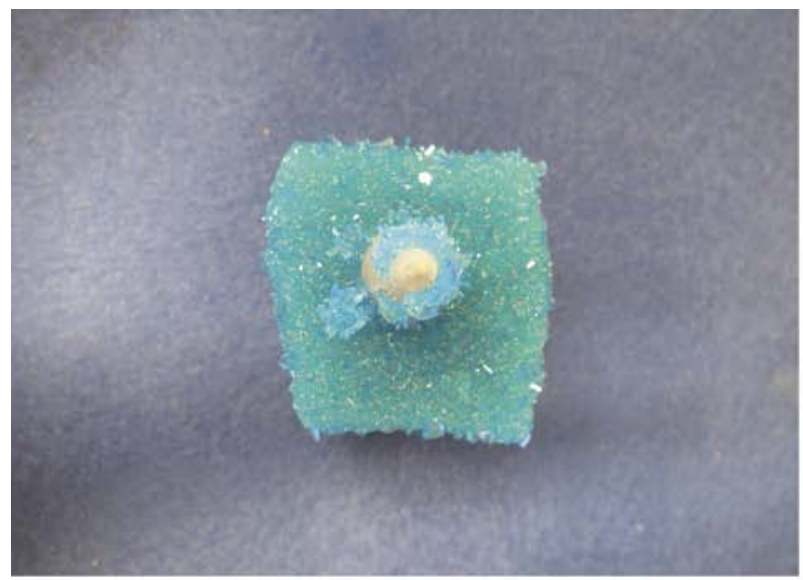

a)

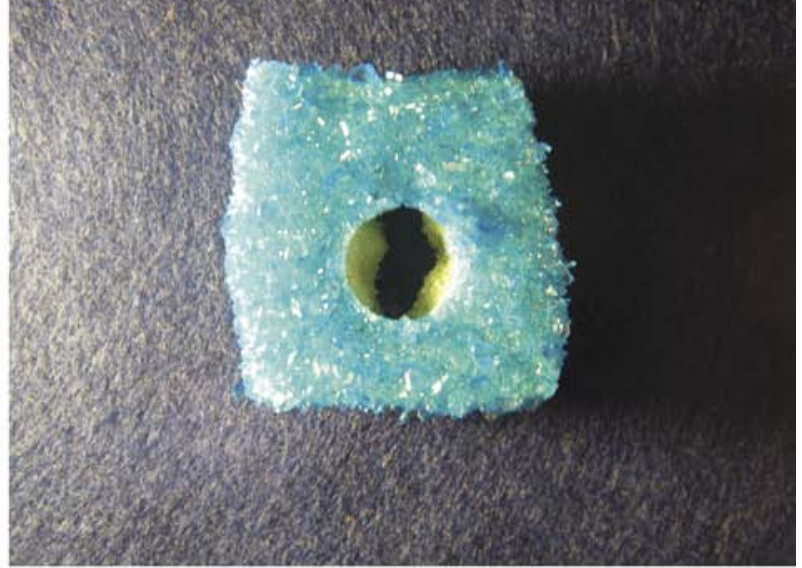

b)

Figure 3. Sponge with aluminum cone after crystallization of cupric sulphate pentahydrate (a) and after removal of aluminum cone (b)

3. The cone was inserted into the sponge (Figure $2 b$ ).

4. The cone and sponge were immersed into the saturated cupric sulfate solution.

5. The sponge was squeezed so as to absorb as much saturated cupric sulfate solution as possible.

6. The cone and the sponge were left in the saturated solution for 24 hours.

7. The cone and the sponge were removed from the solution (Figure 3a).

8. The cone was removed from the sponge.

It can be seen from Figure $3 b$ that the hole is virtually well preserved. For a better comparison, Figure 4 reveals different parts of the sponge with/ without cupric sulfate pentahydrate crystals. The

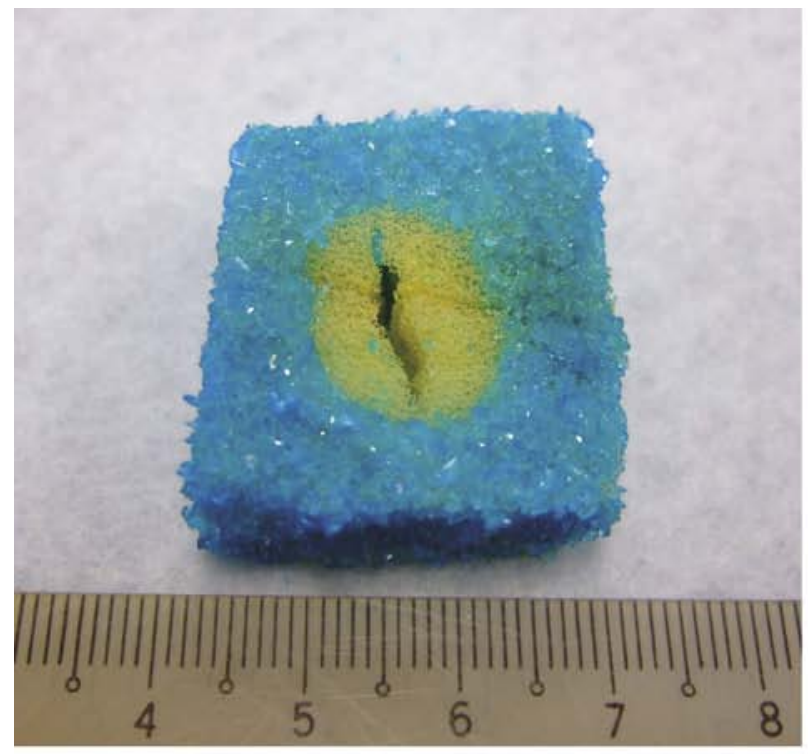

a) part with cupric sulfate pentahydrate crystals is solid and stiff, while the part without the crystals is porous, soft and elastic.

Figure 5 shows the cyclic uniaxial compression testing results of both the original sponge and the sponge/cupric sulfate pentahydrate hybrid. It can be seen that the stiffness of sponge/cupric sulfate pentahydrate hybrid is significantly higher, which indicates that after crystallization, cupric sulfate pentahydrate is strong enough to retain the shape of the deformed sponge, i.e., shape recovery is largely prevented so that the hole can be largely maintained.

It can also be noticed that remarkable residual strain occurs in the original sponge upon loaded to about $12.5 \%$ compressive strain. As such, the elasticity of

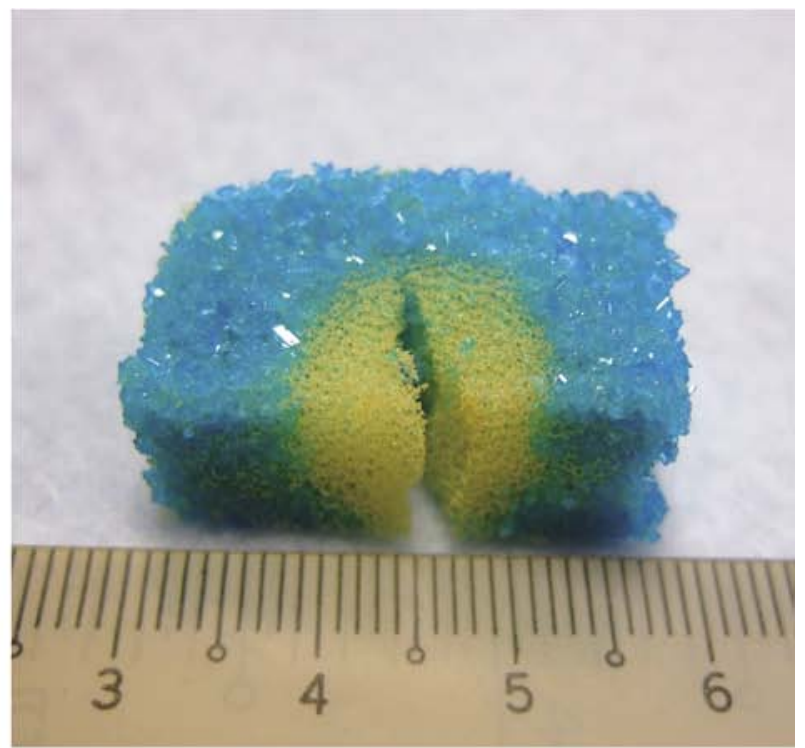

b)

Figure 4. Comparison of parts with/without cupric sulphate pentahydrate in a sponge (a) and a sectional view (b). The unit indicated in the ruler is in $\mathrm{cm}$. 


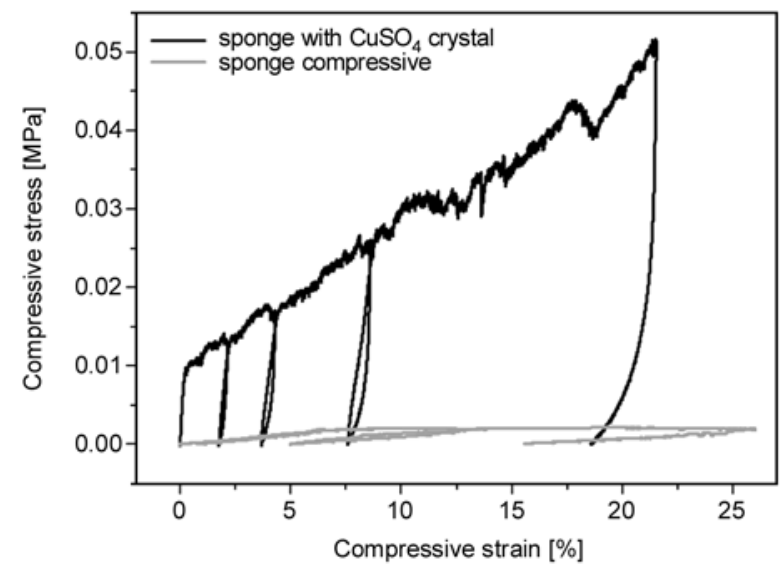

Figure 5. Stress vs. strain curves of sponge and sponge with cupric sulphate pentahydrate $\left(\mathrm{CuSO}_{4}\right)$ in cyclic uniaxial compression tests

the sponge is limited. This is consistent with the observed residual crack after penetration shown in Figure 2c.

In the second step, the sponge (Figure $3 b$ ) was immersed into room temperature water. Figure 6 presents the snapshots of the recovery (hole clo-

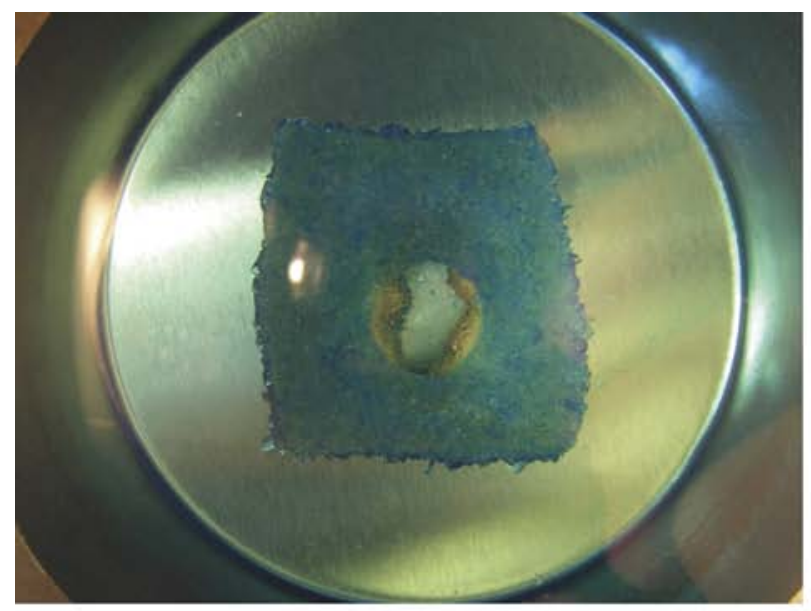

a)

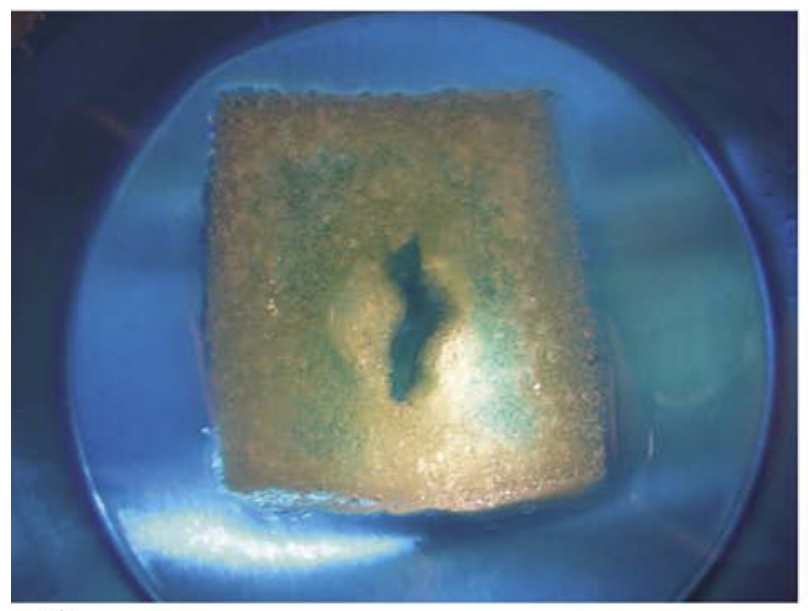

c) sure) sequence. As cupric sulfate pentahydrate gradually dissolved in the water, the color of the sponge changed from dark blue back to its original color, and the hole closed gradually due to the release of the elastic energy stored in the pre-deformed sponge. After about 6 minutes, the hole was largely closed.

\subsection{Silicone/sodium acetate trihydrate hybrid}

Silicone has become a widely used material for a variety of applications, mainly due to its excellent biocompatibility and elasticity. Sylgard ${ }^{\circledR} 184$ silicone elastomer (from Dow Corning) was used as the elastic matrix, and sodium acetate trihydrate (from Sigma-Aldrich) as the transition inclusion in this part of study. The as-received silicone came in two parts, i.e., liquid silicone base and curing agent. After mixing in a ratio of 10:1, curing can be done either at room temperature or at high temperatures (for a shorter curing time). The cured silicone is transparent, rubber-like and mechanically/chemically stable from -40 to $270^{\circ} \mathrm{C}$.

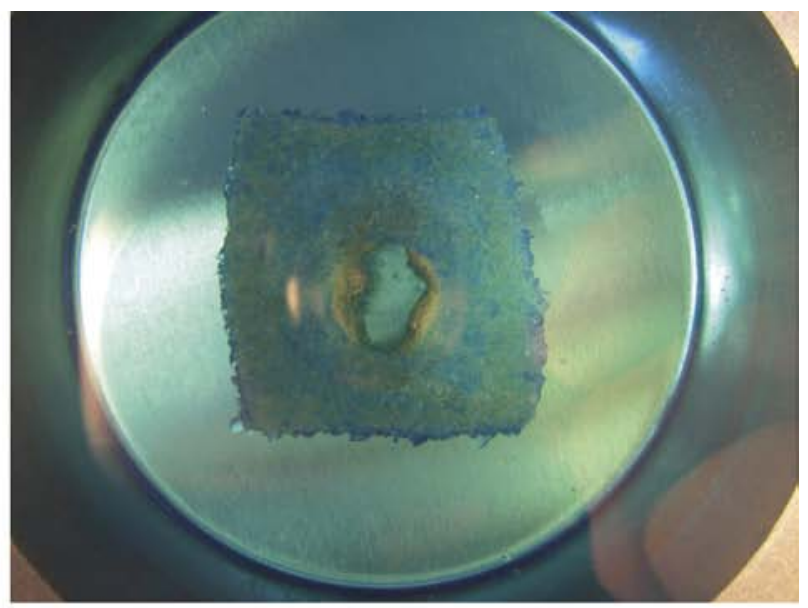

b)

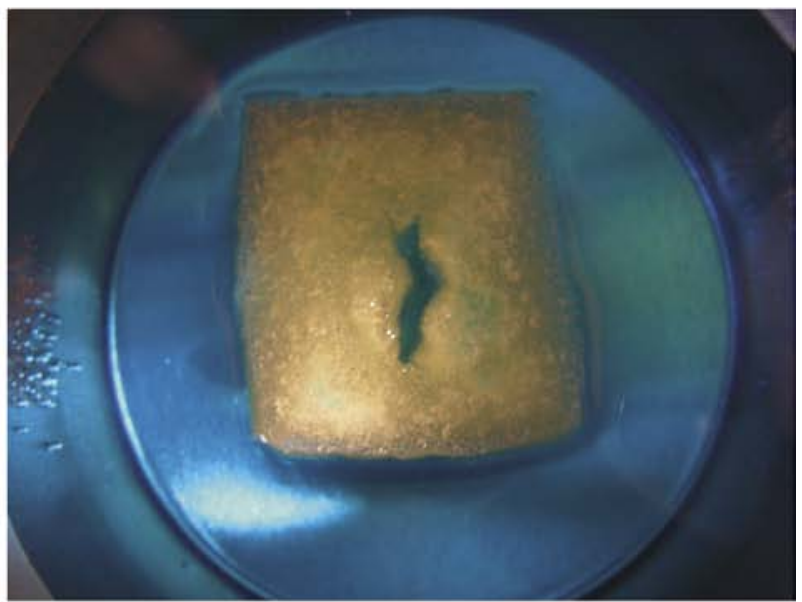

d)

Figure 6. Shape recovery (hole closure) of sponge/cupric sulphate pentahydrate hybrid upon immersing into room temperature water $(\mathrm{a}-\mathrm{d})$ 
Different from cupric sulfate pentahydrate, upon heating sodium acetate trihydrate starts to melt at about $60^{\circ} \mathrm{C}$ due to the transition of sodium acetate trihydrate $\left(\mathrm{C}_{2} \mathrm{H}_{3} \mathrm{NaO}_{2} \cdot 3 \mathrm{H}_{2} \mathrm{O}\right)$ into sodium acetate anhydrous $\left(\mathrm{C}_{2} \mathrm{H}_{3} \mathrm{NaO}_{2}\right)$ and water $\left(3 \mathrm{H}_{2} \mathrm{O}\right)$ (sodium acetate dissolved in this $3 \mathrm{H}_{2} \mathrm{O}$ ) (refer to Figure 7). At above $85^{\circ} \mathrm{C}$, a full liquid state is reached. Hence, sodium acetate liquid can be mixed with liquid silicone base at above $85^{\circ} \mathrm{C}$. After cooling back to room temperature, curing agent can be added in for solidifying the silicone. As an alternative, at room temperature sodium acetate trihydrate can be ground into tiny powders and the powders can then be mixed directly into the silicone. In our experiment, the latter was followed as this approach is more

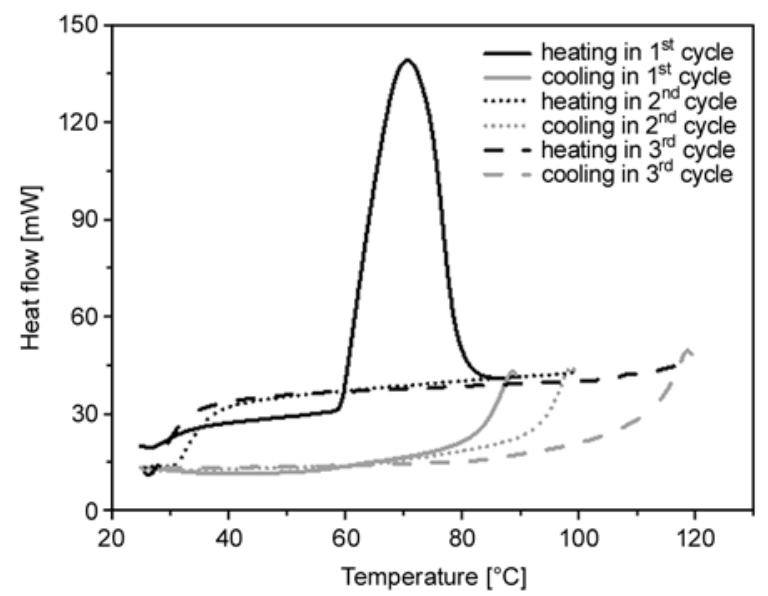

Figure 7. Result of differential scanning calorimeter test of sodium acetate trihydrate

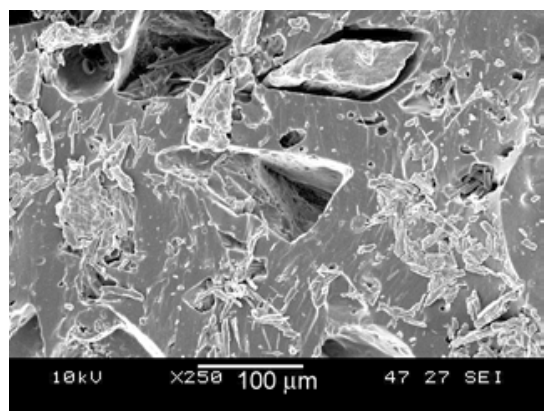

Figure 8. Typical SEM image showing the dispersion of sodium acetate trihydrate (at $50 \mathrm{vol} \%$ ) within silicone convenient. Figure 8 is a typical SEM image, which reveals the dispersion of sodium acetate trihydrate within silicone matrix. It can be seen clearly that tiny sodium acetate trihydrate crystals actually have a tendency towards forming aggregates. A closer look reveals that these aggregates are not fully isolated from each other but connected by some separate crystals.

In order to program the silicone/sodium acetate trihydrate hybrid into a temporary shape, a piece of this hybrid sample (strip shape, with $60 \mathrm{vol} \%$ of sodium acetate trihydrate) was heated to above the melting temperature of sodium acetate trihydrate and then bent into a U-shape. After cooling back to room temperature, sodium acetate trihydrate recrystallized. Hence, apart from a slight elastic recovery, the deformed shape was largely preserved (becoming V-shape) after the constraint was removed. Thermally induced shape recovery (i.e., the thermo-responsive shape memory effect) was examined by immersing the distorted hybrid sample into hot water. As revealed in Figure 9, the sample recovered its original flat strip shape in $18 \mathrm{~s}$ in $72.5^{\circ} \mathrm{C}$ water.

To verify the water-responsive feature, a hybrid ring (with $50 \mathrm{vol} \%$ of sodium acetate trihydrate) was prepared (refer to Figure 10, original). The ring was heated to above the melting temperature of sodium acetate trihydrate and deformed into a Yshape. After cooling back to room temperature, the deformed shape was mainly retained. Subsequently, the deformed ring was immersed into room temperature water. Figure 10 reveals the sequence that the ring gradually recovered its original shape after 50 hours of immersion.

\subsection{Discussion}

Two approaches have been demonstrated to fabricate water-responsive shape memory hybrids. In the first approach, the temporary shape was achieved by means of re-crystallization of the inclusion

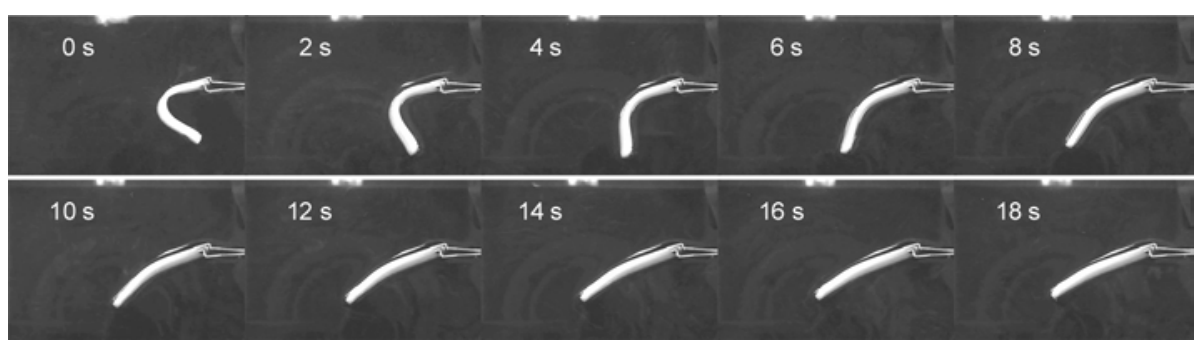

Figure 9. Shape recovery of a strip hybrid ( $60 \mathrm{vol} \%$ of sodium acetate trihydrate) upon immersing into $72.5^{\circ} \mathrm{C}$ water 

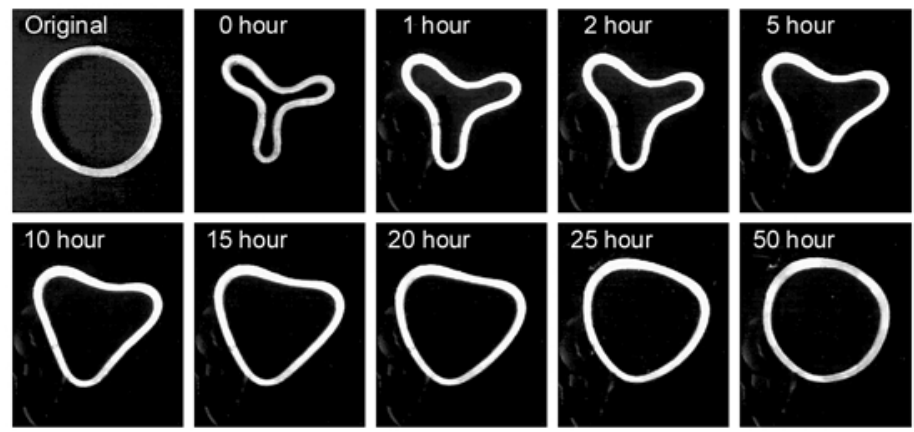

Figure 10. Shape recovery of a hybrid ring (50 vol\% of sodium acetate trihydrate) upon immersing into room temperature water

material inside the deformed porous matrix. As the porous matrix is a cured polymer, the possible chemical interaction between the inclusion and matrix is minimized. In the second approach, the temporary shape was achieved by heating the hybrid to the melting temperature of the inclusion, deforming the hybrid and then followed by cooling it back to the room temperature. As we can see, by selecting a proper processing procedure, we can limit, if not totally avoid, the possible chemical interaction between the matrix and the inclusion.

Depending on the type of the inclusion, the porosity of the matrix, the shape of inclusion (closed cell or open cell), and/or the permeability of the matrix, the speed of shape recovery can be controlled.

As compared with the traditional approaches to fabricate water-responsive shape memory polymers (e.g., [25]), the proposed hybrid concept is more convenient and versatile. For instance, the types of inclusion materials are not limited. Hence, medicine can even be used as the inclusion for drugrelease $[26,27]$. Retractable eluting shape memory stent, which can release drug gradually and eventually shrink its size for easy removal, could be a potential application area.

After the materials for the matrix and inclusion are selected, one can easily predict the properties and behaviors of the resulted hybrid, i.e., the hybrid can be designed following a standard approach as in the design of a conventional composite material. This is another major advantage of this hybrid concept.

This concept can be easily extended into solventresponsive shape memory hybrids [28], in which a particular chemical is the stimulus to trigger the shape recovery or other means of mechanical action. Consequently, targeted drug release can be more conveniently (or even routinely) realized. Once the hybrid senses a particular chemical from the surrounding environment, it releases the drug automatically $[29,30]$.

\section{Conclusions}

In this paper, the concept of water-responsive shape memory hybrid is proposed. The advantages are demonstrated by the two examples. As we can see, the hybrid concept is versatile and can be easily accessed by those even without much polymer/ chemistry background. Moreover, the performance of the resulted hybrid can be well-predicted in the early design stage. This concept can also be further extended into solvent-responsive shape memory hybrids, which can be routinely realized in a Do-ItYourself manner by almost anyone.

\section{References}

[1] Huang W. M., Ding Z., Wang C. C., Wei J., Zhao Y., Purnawali H.: Shape memory materials. Materials Today, 13, 54-61 (2010). DOI: $10.1016 / \mathrm{S} 1369-7021(10) 70128-0$

[2] Otsuka K., Wayman C.: Shape memory materials. Cambridge University Press, Cambridge (1998).

[3] Miyazaki S., Fu Y. Q., Huang W. M.: Thin film shape memory alloys-fundamentals and device applications. Cambridge University Press, Cambridge (2009).

[4] Entel P., Gruner M. E., Dannenberg A., Siewert M., Nayak S. K., Herper H. C., Buchelnikov V. D.: Fundamental aspects of magnetic shape memory alloys: Insights from ab initio and Monte Carlo studies. Materials Science Forum, 635, 3-12 (2009). DOI: $10.4028 /$ www.scientific.net/MSF.635.3

[5] Rousseau I. A.: Challenges of shape memory polymers: A review of the progress toward overcoming SMP's limitations. Polymer Engineering and Science, 48, 2075-2089 (2008). DOI: $10.1002 /$ pen.21213 
[6] Mather P. T., Luo X., Rousseau I. A.: Shape memory polymer research. Annual Review of Material Research, 39, 445-471 (2009).

DOI: 10.1146/annurev-matsci-082908-145419

[7] Huang W. M., Yang B., Zhao Y., Ding Z.: Thermomoisture responsive polyurethane shape-memory polymer and composites: A review. Journal of Materials Chemistry, 20, 3367-3381 (2010).

DOI: $10.1039 / B 922943 \mathrm{D}$

[8] Xie T.: Tunable polymer multi-shape memory effect. Nature, 464, 267-270 (2010).

DOI: $10.1038 /$ nature 08863

[9] Funakubo H.: Shape memory alloys. Gordon and Breach Science Publishers, New York (1987).

[10] Lendlein A.: Shape-memory polymers. Springer, Heidelberg (2010).

DOI: $10.1007 / 978-3-642-12359-7$

[11] Leng J., Du S.: Shape-memory polymers and multifunctional composites. CRC Press, Boca Raton (2010).

[12] Sun L., Huang W. M.: Thermo/moisture responsive shape-memory polymer for possible surgery/operation inside living cells in future. Materials and Design, 31, 2684-2689 (2010).

DOI: $10.1016 /$ j.matdes.2009.11.036

[13] Huang W. M.: On the selection of shape memory alloys for actuators. Materials and Design, 23, 11-19 (2002). DOI: $10.1016 / \mathrm{S} 0261-3069(01) 00039-5$

[14] Gunes S. I., Jana S. C.: Shape memory polymers and their nanocomposites: A review of science and technology of new multifunctional materials. Journal of Nanoscience and Nanotechnology, 8, 1616-1637 (2008). DOI: $10.1166 /$ jnn.2008.038

[15] Yakacki C. M., Shandas R., Safranski D., Ortega A. M., Sassaman K., Gall K.: Strong, tailored, biocompatible shape-memory polymer networks. Advanced Functional Materials, 18, 2428-2435 (2008).

DOI: $10.1002 / \mathrm{adfm} .200701049$

[16] Xie T., Xiao X., Cheng Y-T.: Revealing triple-shape memory effect by polymer bilayers. Macromolecular Rapid Communications, 30, 1823-1827 (2009). DOI: $10.1002 /$ marc. 200900409

[17] Xie T., Rousseau I. A.: Facile tailoring of thermal transition temperatures of epoxy shape memory polymers. Polymer, 50, 1852-1856 (2009).

DOI: $10.1016 /$ j.polymer.2009.02.035

[18] Nair D. P., Cramer N. B., Scott T. F., Bowman C. N., Shandas R.: Photopolymerized thiol-ene systems as shape memory polymers. Polymer, 51, 4383-4389 (2010).

DOI: $10.1016 /$ j.polymer.2010.07.027

[19] Yang B., Huang W. M., Li C., Li L.: Effects of moisture on the thermomechanical properties of a polyurethane shape memory polymer. Polymer, 47, 1348-1356 (2006).

DOI: 10.1016/j.polymer.2005.12.051
[20] Pretsch T., Müller W. W.: Shape memory poly(ester urethane) with improved hydrolytic stability. Polymer Degradation and Stability, 95, 880-888 (2010).

DOI: $10.1016 /$ j.polymdegradstab.2009.12.020

[21] Lorenzo V., Díaz-Lantada A., Lafont P., Lorenzo-Yustos H., Fonseca C., Acosta J.: Physical ageing of a PUbased shape memory polymer: Influence on their applicability to the development of medical devices. Materials and Design, 30, 2431-2434 (2009).

DOI: $10.1016 / \mathrm{j}$. matdes.2008.10.023

[22] Smith K. E., Parks S. S., Hyjek M. A., Downey S. E., Gall K.: The effect of the glass transition temperature on the toughness of photopolymerizable (meth)acrylate networks under physiological conditions. Polymer, 50, 5112-5123 (2009).

DOI: $10.1016 /$ j.polymer.2009.08.040

[23] Weiss R. A., Izzo E., Mandelbaum S.: New design of shape memory polymers: Mixtures of an elastomeric ionomer anf low molar mass fatty acids and their salts. Macromolecules, 41, 2978-2980 (2008).

DOI: $10.1021 / \mathrm{ma} 8001774$

[24] Huang W. M., Yang B., An L., Li C., Chan Y. S.: Water-driven programmable polyurethane shape memory polymer: Demonstration and mechanism. Applied Physics Letters, 86, 114105/1-114105/3 (2005). DOI: $10.1063 / 1.1880448$

[25] Jung Y. C., So H. H., Cho J. W.: Water-responsive shape memory polyurethane block copolymer modified with polyhedral oligomeric silsesquioxane. Journal of Macromolecular Science Part B: Physics, 45, 453-461(2006).

DOI: 10.1080/00222340600767513

[26] Chen M-C., Chang Y., Liu C-T., Lai W-Y., Peng S-F., Hung Y-W., Tsai H-W., Sung H-W.: The characteristics and in vivo suppression of neointimal formation with sirolimus-eluting polymeric stents. Biomaterials, 30, 79-88 (2009).

DOI: $10.1016 /$ j.biomaterials.2008.09.006

[27] Wischke C., Lendlein A.: Shape-memory polymers as drug carriers - A multifunctional system. Pharmaceutical Research, 27, 527-529 (2010). DOI: $10.1007 / \mathrm{s} 11095-010-0062-5$

[28] Du H., Zhang J.: Solvent induced shape recovery of shape memory polymer based on chemically crosslinked poly(vinyl alcohol). Soft Matter, 6, 3370-3376 (2010). DOI: $10.1039 / \mathrm{b} 922220 \mathrm{k}$

[29] Gall K., Kreiner P., Turner D., Hulse M.: Shape-memory polymers for microelectromechanical systems. Journal of Microelectromechanical Systems, 13, 472483 (2004). DOI: $10.1109 /$ JMEMS.2004.828727

[30] Lee B. R., Baik H. J., Oh N. M., Lee E. S.: Intelligent polymeric nanocarriers responding to physical or biological signals: A new paradigm of cytosolic drug delivery for tumor treatment. Polymers, 2, 86-101 (2010). DOI: $10.3390 /$ polym2020086 